\title{
$\mathrm{TiO}_{2}$ 前驱体微胶囊型缓蚀剂的制备与性能研究
}

\author{
李洪广，间军，汪明球，孟胜皓，杜仕国 \\ (军械工程学院三系, 石家庄 050003)
}

摘 要: 随着火炮初速、膛压、射速的提高, 炮膛的烧蚀磨损逐渐变得严重, 对高效缓蚀剂的需求越来越迫切。本 研究以聚乙烯醇(PVA)为壁材, 在 $\mathrm{W} / \mathrm{O} / \mathrm{W}$ 复乳体系中利用界面交联法制备了 $\mathrm{TiO}_{2}$ 前驱体微胶囊, 探讨了微胶囊的 形成机理。扫描电镜观察表明微胶囊呈现出球形形貌, 分散性好, 囊壁致密、包覆完整, 粒径分析显示其平均粒径 在 $10 \mu \mathrm{m}$ 左右; 红外分析进一步证实微胶囊是由醇醛树脂层包覆 $\mathrm{TiO}_{2}$ 前驱体形成; 热分析表明微胶囊中固体 $\mathrm{TiO}_{2}$ 含量约为 $35 \%$ 。利用烧蚀管试验法测试其降烧蚀性能, 结果表明 $\mathrm{TiO}_{2}$ 前驱体微胶囊型缓蚀剂不影响发射药的燃烧 规律, 在相对于发射药质量 $2.5 \mathrm{wt} \%$ 的添加量下, 降烧蚀效率达到 $35 \%$ 左右。

关 键 词: 烧蚀磨损; 缓蚀剂; $\mathrm{TiO}_{2}$ 前驱体; 微胶囊

中图分类号: TJ307 文献标识码: A

\section{Microcapsulation of $\mathrm{TiO}_{2}$ precursor and Its Performance as Inhibitor of Erosion}

\author{
LI Hong-Guang, YAN Jun, WANG Ming-Qiu, MENG Sheng-Hao, DU Shi-Guo \\ (The 3rd Department of Ordnance Engineering College, Shijiazhuang 050003, China)
}

\begin{abstract}
As erosion and wear of gun bore become increasingly serious with the increase of muzzle velocity, chamber pressure and rate of fire, the demand for their high effective inhibitor is urgent. $\mathrm{TiO}_{2}$ precursor microcapsules with polyvinyl alcohol (PVA) as wall material were prepared by interfacial crosslinking method in W/O/W multiphase emulsion. The microcapsules were characterized by scanning electron microscope (SEM), particle size analyzer, fourier transform infrared spectrometer (FT-IR), thermogravimetric analyzer (TG). The results showed that the microcapsules were dispersive spherical particles at average size of $10 \mu \mathrm{m}$ with integral and dense shell. The microcapsules were composed of about $35 \%$ solid $\mathrm{TiO}_{2}$ and aldol resin layer. Its anti-erosion effect was tested by erosion tube method, and showed that the combustion law of propellant was not affected after application and the anti-erosion efficiency was $35 \%$ when added $2.5 \mathrm{wt} \%$ microcapsules relative to propellant mass.
\end{abstract}

Key words: erosion and wear; inhibitor; $\mathrm{TiO}_{2}$ precursor; microcapsule

现代战争要求火炮具有更高的初速以增大射 程、更高的射速以增强火力，随着火炮初速、膛压、 射速的提高, 炮膛的烧蚀磨损变得十分严重, 大大
缩短了武器的使用寿命。大威力火炮应用新型高能 发射药, 造成火炮身管的寿命成倍地下降 ${ }^{[1]}$ 。在新型 电热化学炮发射过程中, 高能、高密度的发射装药

收稿日期：2014-04-16; 收到修改稿日期：2014-06-18

基金项目: 国家自然科学基金(51272284); 军械工程学院科学研究基金(YJJXM13016)

National Natural Science Foundation of China (51272284); Science Research Foundation of Ordnance Engineering College (YJJXM13016)

作者简介: 李洪广(1988-), 男, 博士研究生. E-mail: qdlhgcy6@126.com

通讯作者: 杜仕国, 教授. E-mail: yan-junjun@263.net 
也将对炮管造成更为严重的烧蚀, 炮管烧蚀也成为 新型电热化学发射技术中的关键而备受关注 ${ }^{[2]}$ 。

针对身管武器烧蚀磨损问题, 国内外专家学者 探索了使用低爆温发射药、内膛涂镀层技术、抗烧 蚀祄管技术、缓蚀剂技术以及复合身管制造技术等 多种措施 ${ }^{[3-9]}$ 。其中, 较实用、有效、被国内外广泛 采用的方法是在装药系统中加入缓蚀剂 ${ }^{[10-11]}$ 。即使 火炮身管采用了内膛镀铬技术，也必须同缓蚀剂技 术配合使用，才能更有效地提高身管使用寿命 ${ }^{[12]}$ 。 因此, 大威力火炮和速射武器的不断发展还将依赖 于缓蚀剂技术的进步 ${ }^{[13]}$ 。

传统的缓蚀剂主要是由二氧化钛、滑石粉、石 蜡、地蜡及其混合物组成 ${ }^{[9,14-15]}$, 多涂覆在纤维布上 以护膛祄里的形式应用。为改进性能与使用效果, 碳酸钙、有机硅、超细滑石粉以及纳米材料等也应

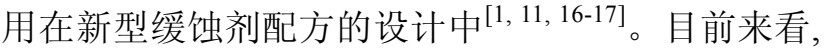
缓蚀剂的结构多为有机载体材料加无机粉体材料的 形式。该型缓蚀剂在满足大威力火炮的基本降烧蚀 要求的同时, 存在用量大、占用装药空间大、内膛 表面分散性较差、局部聚积不完全燃烧残渣，以及 对内弹道性能不良影响增大等问题 ${ }^{[18]}$ 。

微胶囊技术是一种用成膜材料把固体、液体或 气体包覆形成微小粒子的技术, 在各领域有广泛应 用。微胶囊的粒径小、表面积大，有利于分散和燃 烧完全; 无机芯材被包覆于有机壁材内, 可防止其 在爆炸燃烧过程中的聚积; 液态或半固态 $\mathrm{TiO}_{2}$ 前驱 体被包覆转化为自由流动的固体粉末, 可方便使用 和装填。因此, 微胶囊自身的这些特点有可能帮助 解决缓蚀剂在使用中存在的诸多问题。

为此, 本研究探索了新型结构形式的微胶囊 缓蚀剂。设计了以有机树脂为壁材、 $\mathrm{TiO}_{2}$ 前驱体 为芯材的微胶囊型缓蚀剂。并对所制备的微胶囊 进行了表征，采用烧蚀管试验法，测试了其降烧 蚀性能。

\section{1 实验方法}

\section{1 设计思想}

$\mathrm{TiO}_{2}$ 前驱体是由有机钛醇盐, 如钛酸丁酯, 通 过控制水解获得的溶胶体系, 该前驱体在炮膛的高 温、高压环境下会转变形成 $\mathrm{TiO}_{2}$, 形成隔热层, 降 低火药气体对炮管的烧蚀。聚乙烯醇因具有易成膜、 机械性能好、热稳定性高、安全低毒、价格便宜、 使用方便等优点，在微胶囊中得到广泛应用 ${ }^{[19-22]}$; 同时聚乙烯醇的化学成分与石蜡相似，发射过程中 会形成冷的边界层, 起到保护内膛的作用。

\section{2 微胶囊的制备}

将钛酸丁酯、乙酰丙酮按体积比 15:1 溶于无水 乙醇中，剧烈搅拌条件下缓慢滴加去离子水至完全 沉淀, 然后滴加浓度为 $30 \%$ 的硝酸至沉淀消失形成 透明的 $\mathrm{TiO}_{2}$ 溶胶 ${ }^{[23]}$ 。将质量分数为 $50 \%$ 的戊二醛水 溶液加入到上述溶胶中, 摚拌均匀后作为内水相, 内水相中戊二醛的体积分数为 $10 \%$ 。将 Span-80 溶 于环己烷形成油相, 油相中 Span-80 的体积分数为 $5 \%$ 。在 8000 10000 r/min 的乳化速率下, 将内水相加 入油相形成 W/O 乳状液。在 600 800 r/min 的搅拌速 率下, 将上述乳液滴加入质量分数为 $1 \%$ 的 PVA 水 溶液中形成 $\mathrm{W} / \mathrm{O} / \mathrm{W}$ 复相乳液, 于 $40^{\circ} \mathrm{C}$ 下保温 $1 \mathrm{~h}$ 。 离心分离产物, 并用水和乙醇洗涤 3 5 次, 冷冻干 燥后得到微胶囊样品。

\section{3 样品的分析与表征}

样品的形貌由 S-4800 型扫描电子显微镜(SEM, 日本 HITACHI 公司)观察; 粒径分布通过 JL-1155 型激光粒度分布仪(成都精细粉体测试设备有限公 司)测试; 结构通过 VERTEX 70 型傅立叶变换红外 光谱仪(德国 Bruker 公司, $\mathrm{KBr}$ 压片)测试; 利用 TGA 7 型热重分析仪(美国 PerkinElmer 公司)研究样品热 性能; 利用 CDR-4P 型差式扫描量热仪(上海天平厂, 静态空气气氛, 升温速率为 $5{ }^{\circ} \mathrm{C} / \mathrm{min}, \alpha-\mathrm{Al}_{2} \mathrm{O}_{3}$ 为参 比)测定样品的 DSC 曲线。

\section{4 降烧蚀性能试验}

采用烧蚀管失重法检测微胶囊的降烧蚀性能, 烧蚀管材料为 $45^{\#}$ 钢, 发射药的装药量为 $11.8 \mathrm{~g}$, 燃 烧环境为半密闭爆发器, 点火药为 $2^{\#} \mathrm{NC}$ 。选用的发 射药为含有硝化甘油 $(\mathrm{NG})$ 、烧蚀性强的双基药。利 用 DSC 法测试了微胶囊与该型发射药的相容性, 测 试按照 GJB772A-97 方法 502.1 条进行。实验条件 为: 铝坩埚, 静态空气气氛, 升温速率为 $5{ }^{\circ} \mathrm{C} / \mathrm{min}$, 试样量均为 $0.7 \mathrm{mg}, \alpha-\mathrm{Al}_{2} \mathrm{O}_{3}$ 为参比。

\section{2 结果与讨论}

\section{1 微胶囊的形貌及形成过程}

$\mathrm{W} / \mathrm{O} / \mathrm{W}$ 复乳体系中制备微胶囊的工艺流程如 图 1 所示。在微胶囊制备过程中发现, W/O 孚液的 稳定性是制备分散均匀微胶囊的关键。图 2 和图 3 分别为实验条件下所制备的 $\mathrm{W} / \mathrm{O}$ 乳液的显微镜照 片及微胶囊的 SEM 照片。由图可见, W/O 乳液液滴 的大小与形状基本上决定了微胶囊的大小与形貌。

由图 2 可见, 所制备的 W/O 乳液液滴的粒径均 一、分散性好; 在此基础上制备出的微胶囊呈现出规 


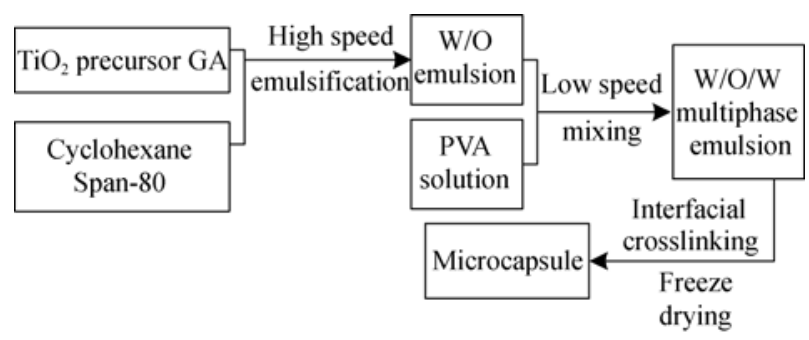

图 1 微胶囊的制备工艺流程

Fig. 1 Preparing process of microcapsules

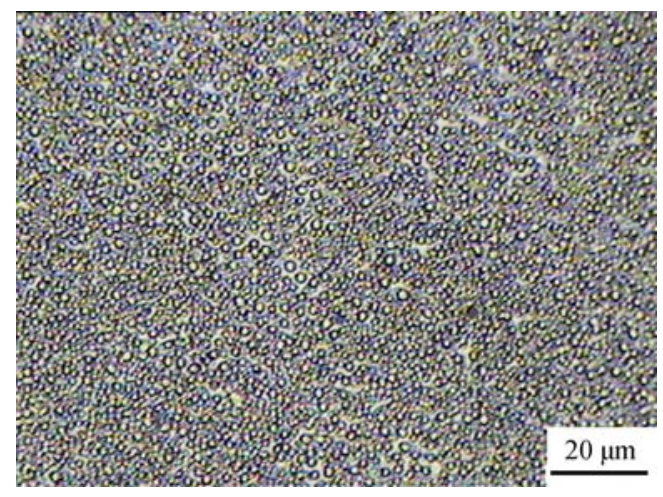

图 2 稳定的 $\mathrm{W} / \mathrm{O}$ 乳液的显微镜照片

Fig. 2 Microscope graph of stabled W/O emulsion
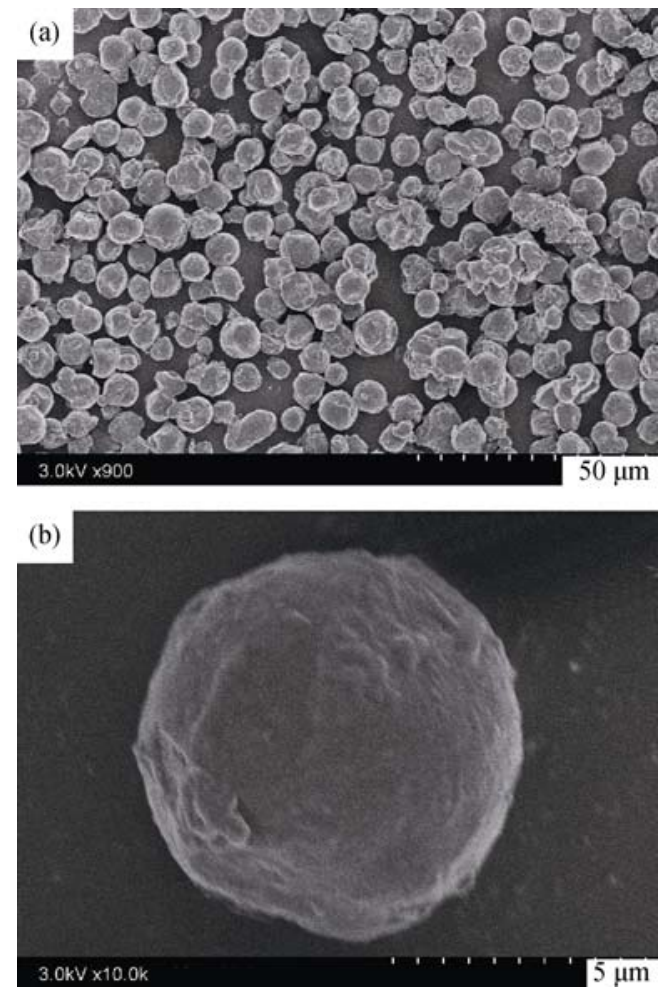

图 3 微胶囊的 SEM 照片

Fig. 3 SEM images of microcapsules

整的球形形貌，分散性好且粒径均匀，囊壁致密，包 覆完整且无明显的孔隙。图 4 为微胶囊的粒度分布曲
线，结果表明微胶囊的粒径分布范围在2 20 $\mu \mathrm{m}$ 之间， 其平均粒径为 $10 \mu \mathrm{m}$ 。

在 $\mathrm{W} / \mathrm{O} / \mathrm{W}$ 复相乳液中, 交联剂 $\mathrm{GA}$ 由内水相向 外水相扩散到油相与外水相的界面, 并与吸附在相 界面的 PVA 发生缩醛反应, 进而沉积在相界面完成 对 $\mathrm{TiO}_{2}$ 前驱体的包覆形成微胶囊。油水界面发生缩 醛化反应的界面交联过程如图 5 所示。

\subsection{FT-IR 分析}

图 6 为样品的 FT-IR 图谱。图中的醇醛树脂由 PVA 与 GA 在水溶液中直接发生交联反应生成, $\mathrm{TiO}_{2}$ 是由微胶囊样品在 $800{ }^{\circ} \mathrm{C}$ 下煅烧后形成。

从醇醛树脂的红外光谱曲线(a)可见, $3440 \mathrm{~cm}^{-1}$ 和 $1378 \mathrm{~cm}^{-1}$ 处的吸收峰为 PVA 中未缩醛化的-OH 的伸 缩振动与弯曲振动; $2928 \mathrm{~cm}^{-1}$ 和 $2857 \mathrm{~cm}^{-1}$ 处为 $-\mathrm{CH}_{2}$ 的伸缩振动峰; $1640 \mathrm{~cm}^{-1}$ 处为 $\mathrm{H}-\mathrm{O}-\mathrm{H}$ 的弯曲振动峰; $1740 \mathrm{~cm}^{-1}$ 处为 $-\mathrm{CHO}$ 上的羰基的吸收峰, 表明醇醛树 脂中含有未反应的醛基; $1140 \mathrm{~cm}^{-1}$ 处的吸收峰为缩醛 反应生成的 $\mathrm{C}-\mathrm{O}-\mathrm{C}-\mathrm{O}-\mathrm{C}$ 的伸缩振动峰, 说明 $\mathrm{PVA}$ 与 $\mathrm{GA}$ 发生了交联反应。从 $\mathrm{TiO}_{2}$ 的曲线(c) 可知, 400 $800 \mathrm{~cm}^{-1}$ 间的宽吸收峰是由 $\mathrm{TiO}_{2}$ 中 $\mathrm{Ti}-\mathrm{O}$ 的伸缩振 动和 $\mathrm{Ti}-\mathrm{O}-\mathrm{Ti}$ 的弯曲振动引起 ${ }^{[24]}$ 。与醇醛树脂的红 外曲线(a)对比, 微胶囊的红外曲线(b)中, $1140 \mathrm{~cm}^{-1}$ 处的吸收峰在 1090 1173 $\mathrm{cm}^{-1}$ 范围内分裂为 3 个峰, 这是由 $\mathrm{C}-\mathrm{O}-\mathrm{C}-\mathrm{O}-\mathrm{C}$ 的伸缩振动发生偶合导致, 说明 微胶囊制备过程中 PVA 与 GA 的交联反应更充分; 而 400 $800 \mathrm{~cm}^{-1}$ 范围内出现的宽吸收峰对应于 $\mathrm{TiO}_{2}$ 红外 曲线(c)中的 Ti-O 的伸缩振动和 $\mathrm{Ti}-\mathrm{O}-\mathrm{Ti}$ 的弯曲振动, 说明 $\mathrm{TiO}_{2}$ 前驱体已被包覆在微胶囊中。这与 SEM 对 微胶囊所观察到的结果相对应(图 3), 进一步说明了 微胶囊是由醇醛树脂层包覆 $\mathrm{TiO}_{2}$ 前驱体所形成。

\subsection{TG/DTG 分析}

醇醛树脂和微胶囊样品的 TG/DTG 曲线如图 7 所示。由图 7 可知, $250^{\circ} \mathrm{C}$ 以前, 醇醛树脂基本没有 失重, 醇醛树脂的热分解主要发生在 $250 \sim 500^{\circ} \mathrm{C}$ 之 间。微胶囊在 $250^{\circ} \mathrm{C}$ 以前失重约为 $10 \%$, 这是由微 胶囊内的吸附水及其他低沸点物质(如少量的溶 剂、油相等)的挥发所致。250 500 ${ }^{\circ} \mathrm{C}$ 之间微胶囊 样品的失重约为 $55 \%$, 此温度区间内包括囊壁材 料醇醛树脂的热分解和芯材 $\mathrm{TiO}_{2}$ 前驱体的失重。 微胶囊热分析曲线中 $380 \sim 450{ }^{\circ} \mathrm{C}$ 之间较大的失重 峰主要为 $\mathrm{TiO}_{2}$ 前驱体向 $\mathrm{TiO}_{2}$ 转变过程, 由前驱体 内的结构水和醇的挥发所致。 $500^{\circ} \mathrm{C}$ 以上微胶囊样 品基本无失重, 其总失重约为 $65 \%$, 由此推知微胶 囊样品中固体 $\mathrm{TiO}_{2}$ 的含量约为 $35 \%$ 。 


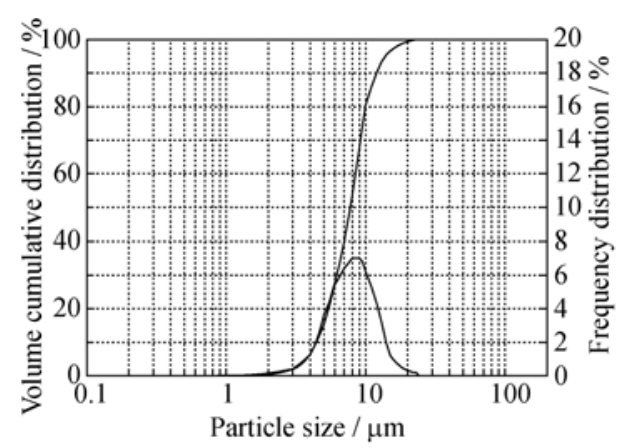

图 4 微胶囊的粒径分布

Fig. 4 Particle size distribution of microcapsules

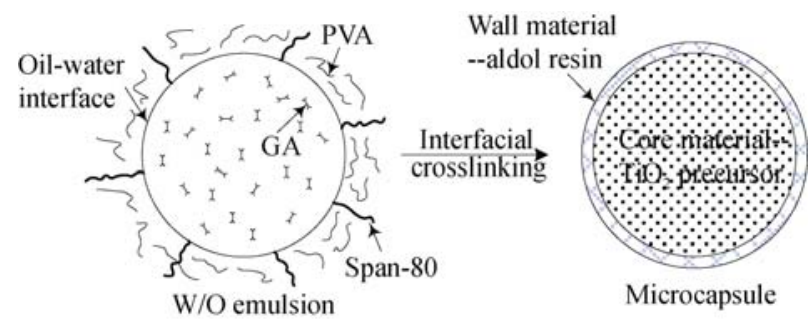

图 5 界面交联过程示意图

Fig. 5 Process of interfacial crosslinking

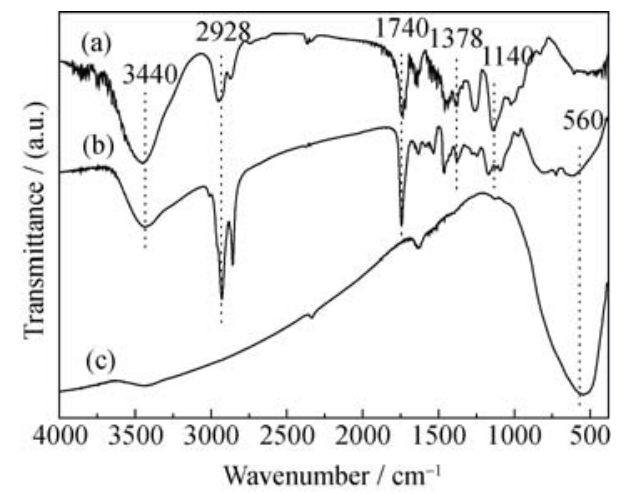

图 6 样品的红外光谱图

Fig. 6 FT-IR spectra of samples

(a) Aldol resin; (b) Microcapsule; (c) $\mathrm{TiO}_{2}$

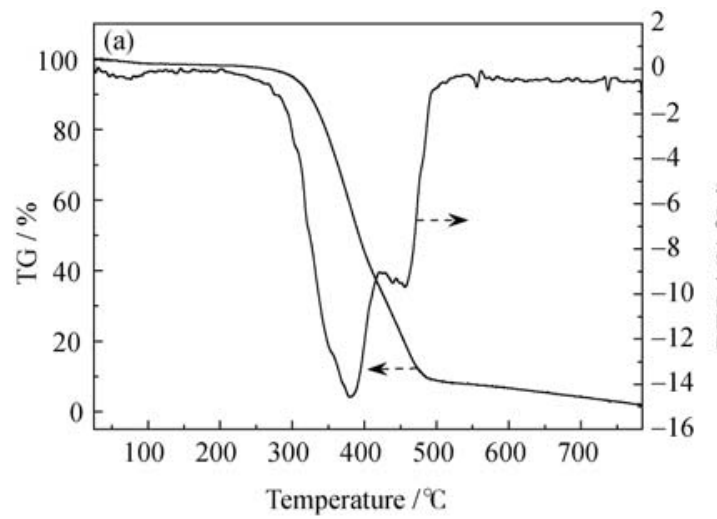

\section{4 烧蚀试验结果}

烧蚀管失重法是一种利用半密闭爆发器试验装 置，以烧蚀管经烧蚀前后的失重量为指标检验缓蚀 剂降烧蚀效果的方法。烧蚀管的烧蚀程度与发射药 的爆温以及所受的火药气体的压力相关, 故试验中 保持发射药的装药量不变。将微胶囊缓蚀剂用同样 大小的可燃纸包住, 并置于发射药的前端, 试验烧 蚀量及平均压力是 3 发的平均值。

烧蚀试验前用 DSC 法测试了微胶囊与发射药 的相容性。微胶囊、发射药及其质量比为 $1: 1$ 混合 体系的 DSC 分析结果如图 8 所示。由图 8 可以看 出, 对于单一组分, 发射药的分解峰值温度为 $196.0^{\circ} \mathrm{C}$, 微胶囊的分解峰值温度为 $305.6^{\circ} \mathrm{C}$ 。微胶 囊与发射药混合后, 微胶囊/发射药二元体系的第 一分解峰值温度为 $196.1^{\circ} \mathrm{C}$, 将发射药的分解吸热 峰值温度推迟了 $0.1^{\circ} \mathrm{C}$, 表明微胶囊与发射药具有 良好的相容性。

烧蚀试验中，对微胶囊在 3 个不同装填量下的 降烧蚀性能进行了测试。加入微胶囊缓蚀剂后半密 闭爆发器的 $P-t$ 曲线与纯发射药的 $P-t$ 曲线非常接近 (图 9), 所以加入缓蚀剂后没有影响发射药的燃烧 规律。从试验结果(表 1)来看: 1) 与空白试验对比, 微胶囊型缓蚀剂表现出了较好的降烧蚀效果, 在 $245 \mathrm{MPa}$ 压力水平下，降烧蚀率达 35\%左右，与相 同压力水平下的有机无机复合缓蚀剂 ${ }^{[11]}$ 相比, 提高 了约 9 个百分点。2) 微胶囊缓蚀剂在较少的装填量 (试验中为 $0.3 \mathrm{~g}$, 相对发射药用量为 $2.5 \mathrm{wt} \%$ ) 时, 就 表现出很好的降烧蚀效果, 符合高效缓蚀剂的要求; 当装填量增至 $0.5 \mathrm{~g}$ 时, 虽然降烧蚀率有所提高, 但 其压力也有所降低; 继续增加装填量至 $0.8 \mathrm{~g}$, 降烧 蚀率明显下降。

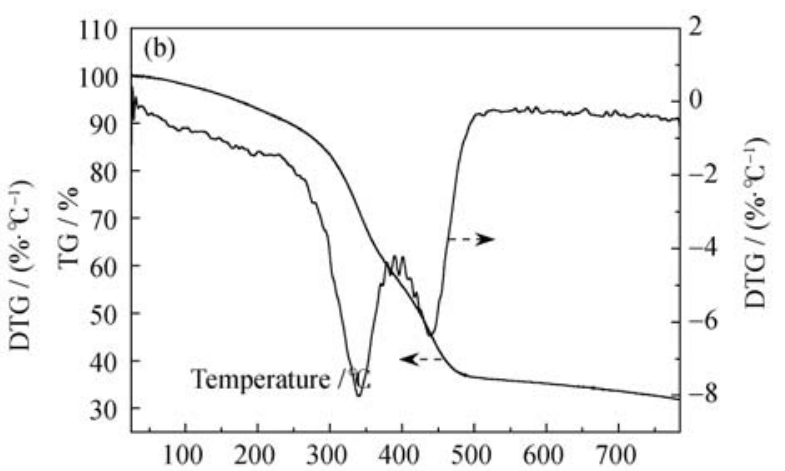

图 7 样品的 TG/DTG 曲线

Fig. 7 TG/DTG curves of samples

(a) Aldol resin; (b) Microcapsule 


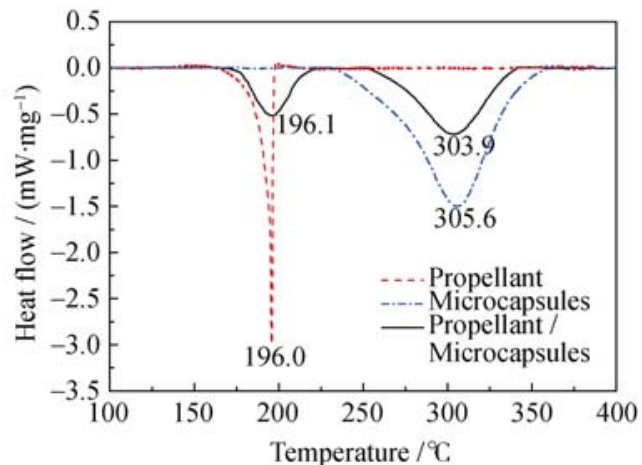

图 8 微胶囊、发射药和微胶囊/发射药混合物的 DSC 曲线 Fig. 8 DSC curves of microcapsules and their mixture with propellant

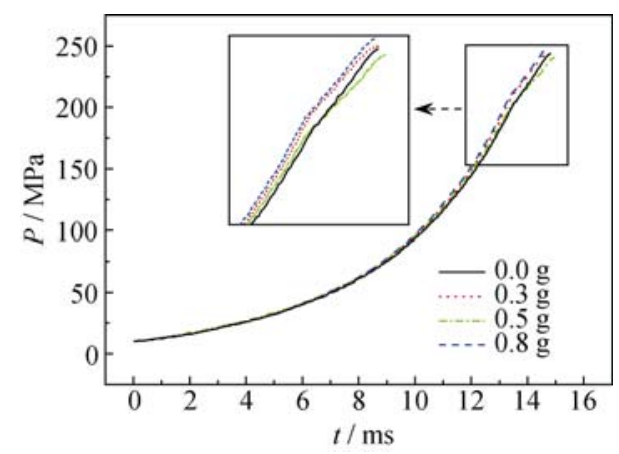

图 9 不同试验条件下的 $P-t$ 曲线

Fig. 9 - $t$ curves at different conditions

表 1 烧蚀试验结果

Table 1 The results of erosion test

\begin{tabular}{ccccc}
\hline $\begin{array}{c}\text { Propellant } \\
\text { mass/g }\end{array}$ & $\begin{array}{c}\text { Inhibitor } \\
\text { mass/g }\end{array}$ & $\begin{array}{c}\text { Average } \\
\text { pressure/ MPa }\end{array}$ & $\begin{array}{c}\text { Amount of } \\
\text { erosion/ mg }\end{array}$ & $\begin{array}{c}\text { Erosion-reducing } \\
\text { efficiency/ \% }\end{array}$ \\
\hline 11.8 & - & 245.2 & 399.7 & - \\
11.8 & 0.3 & 247.9 & 260.9 & 34.7 \\
11.8 & 0.5 & 242.9 & 256.8 & 35.8 \\
11.8 & 0.8 & 248.7 & 289.7 & 27.5 \\
\hline
\end{tabular}

\section{5 降烧蚀机理分析}

由于在半密闭爆发器试验条件下难以收集微胶 囊样品经过爆炸然烧过程后形成的产物, 故将微胶 囊在常压高温条件下煅烧一定时间，收集裧烧后的 产物并观察其形貌。微胶囊样品经常压高温煅烧后 所得产物形貌与实际试验情况有一定差别, 但分析 观察其形貌有助于分析其降烧蚀机理。微胶囊在两 种升温条件下煅烧, 一是将微胶囊在 $5{ }^{\circ} \mathrm{C} / \mathrm{min}$ 的升 温速率下升温至 $800^{\circ} \mathrm{C}$ 并保温 $10 \mathrm{~min}$, 一是将微胶 囊直接在 $800^{\circ} \mathrm{C}$ 下煅烧 $10 \mathrm{~min}$, 煅烧后形成产物的 扫描电镜照片如图 10 所示。

$\mathrm{TiO}_{2}$ 前驱体微胶囊型缓蚀剂具有较高降烧蚀效 率的机理可从以下几方面作出推测:

1) 微胶囊的平均粒径较小, 约 $10 \mu \mathrm{m}$ 左右, 在 短暂的发射过程中可以与火药气体充分作用, 更高 效地发挥降烧蚀作用。
2) 囊壁聚乙烯醇是一种较好的缓蚀材料，能 够为膛壁提供冷边界层; 而芯材 $\mathrm{TiO}_{2}$ 前驱体在高温 下向 $\mathrm{TiO}_{2}$ 的转变过程, 如吸附水和结合水的蒸发以 及 $\mathrm{TiO}_{2}$ 自身的晶型转变等, 可以吸收大量的热, 增 强了冷边界层效应，降低了内膛表面温度，从而减 少了火药气体对身管内膛的烧蚀。

3) 由图 10 可见，微胶囊经缓慢升温炦烧后, 芯材 $\mathrm{TiO}_{2}$ 前驱体形成了结构规整的微球(图 10(a)); 而经过高温直接陆烧后, 形成了结构松散的微球以 及纳米粒子(图 10(b)和(c))。微胶囊经高温单因素的 作用，结构强度较低的 $\mathrm{TiO}_{2}$ 前驱体形成了结构松散 的微球和纳米小颗粒，而在瞬时、高温、高压的爆 炸过程中, 微胶囊的芯材要经历高温、高压两个因 素的冲击，且爆炸过程中的冲击波和震动本身有使 物质震荡、使之松散的力量, 由此推断, 结构强度较 低的 $\mathrm{TiO}_{2}$ 前驱体在爆炸过程中形成纳米
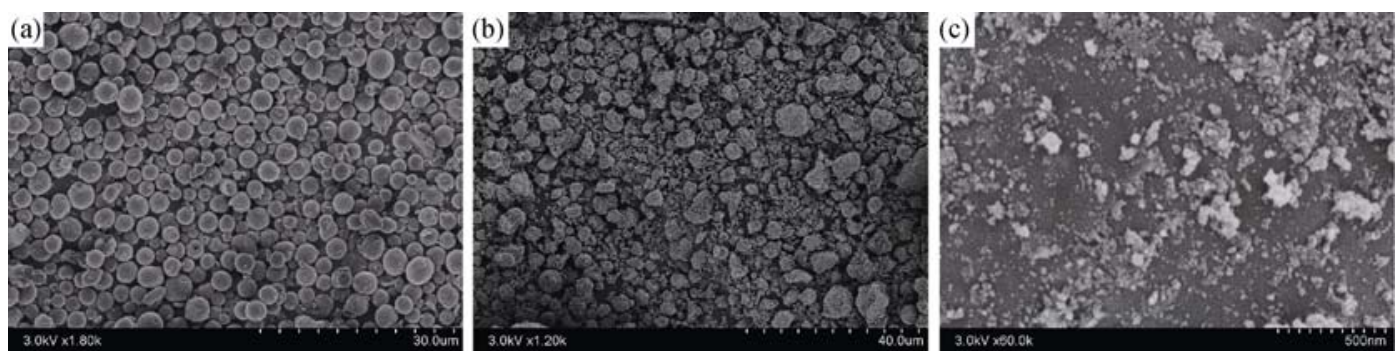

图 10 微胶囊样品煅烧后的扫描电镜照片

Fig. 10 SEM images of microcapsules after calcined at $800^{\circ} \mathrm{C}$ (increased from normal temperature at $\left.5{ }^{\circ} \mathrm{C} / \mathrm{min}\right)(\mathrm{a})$ and directly at $800^{\circ} \mathrm{C}(\mathrm{b}, \mathrm{c})$ for $10 \mathrm{~min}$ 
粒子的机率增大。而纳米粒子优越的小尺寸效应、 高的比表面积更容易使 $\mathrm{TiO}_{2}$ 与火药气体充分接触 发挥自身的降烧蚀作用, 也更容易沉积在内膛表面 形成保护层, 减少火药气体的热、化学烧蚀及机械 磨损。同时, 分散的纳米 $\mathrm{TiO}_{2}$ 粒子也具有润滑、修 复表面等效应。

4) 微胶囊的装填量增加至 $0.8 \mathrm{~g}$, 降烧蚀率明显 下降, 这可能是由以下原因造成: a) 微胶囊的装填量 增大, 发射过程中不能完全燃烧(该条件下半密闭爆 发器腔内存在可见的残渣), 影响了其降烧蚀的效果; b) 装填量增大, 微胶囊燃烧后产生的无机粒子增多, 而单位体积内的纳米粒子增多会引起团聚, 加剧了对 烧蚀管的冲蚀 ${ }^{[25]}$, 从而使烧蚀量增大。

\section{3 结论}

1) 在 $\mathrm{W} / \mathrm{O} / \mathrm{W}$ 复相乳液中, 利用界面交联法制 备了粒径在 $10 \mu \mathrm{m}$ 左右的 $\mathrm{TiO}_{2}$ 前驱体微胶囊。微胶 囊的包覆完整且分散性好, 微胶囊中固体 $\mathrm{TiO}_{2}$ 的含 量约为 $35 \%$ 。

2) DSC 方法测试表明, 微胶囊与试验用双基发 射药的相容性好。

3) $\mathrm{TiO}_{2}$ 前驱体微胶囊是一种高效的缓蚀剂, 在 相对发射药 $2.5 \mathrm{wt} \%$ 的添加量、 $247 \mathrm{MPa}$ 的平均压力 水平下，降烧蚀效率达 $35 \%$ 左右。

\section{参考文献:}

[1] HU SHAN-BAO. Study on Microcapsule Technology of Borewear Reducing Additive. Nanjing: Master Thesis of Nanjing University of Science \& Technology, 2008.

[2] WANG BAO-SHENG. Research for Gun Barrels Anti-erosion Materials and Technologies. GF94582, Beijing: the 210 Research Institute of China Ordnance Industries Group, 2012: 1-69.

[3] CHEN YONG-CAI, SONG QIU-ZHI, WANG JIAN-ZHONG. New technologies to extend the erosion life of gun barrel. Acta Armamentarii, 2006, 27(2): 330-334.

[4] LI DA-YONG, GAO SHOU-ZHEN, LUO CHANG-HONG, et al. Research on renovating the abrasion and ablation of the gun barrel bore. Journal of Gun Launch \& Control, 2012(1): 47-50.

[5] SWAB J J, DANNA D, LEVERITT C, et al. Evaluation of a Foreign Silicon Nitride as a Potential Gun Barrel Liner. ARL-TR-5146, Maryland: U.S. Army Research Laboratory, 2010: 1-18.

[6] LEVY R A. Investigation of Chemically Vapor Deposited Tantalum for Medium Caliber Gun Barrel Protection. OMB No.0704- 0188, New Jersey: New Jersey Institute of Technology, 2008: 1-35.

[7] STORM R, WITHERS J C, RAMOS R, et al. Fabrication of $\mathrm{Si}_{3} \mathrm{~N}_{4}$ gun barrel liners for very high temperature erosion resistant gun barrels. Materials and Manufacturing Processes, 2012, 27(8): 875-877.

[8] SONG QIU-ZHI, DUO YING-XIAN. Influence of a nanomaterial on propellant and the function of a nanomaterial for increasing the service life of a gun barrel. Journal of Harbin Engineering University, 2011, 32(9): 1173-1178.

[9] JOHNSTON I A. Understanding and Predicting Gun Barrel Erosion. DSTO-TR-1757, Edinburgh: Defence Science and Technology Organisation, 2005: 1-37.

[10] GE CHAO-HUI, LIANG XI-YAO. Application Research of a New Ammunition Inhibitor in Armor-piercer. Symposium of the Professional Committee of Tanks and Armored Vehicles of China Ordnance Society, Urumqi, 2006: 318-321.

[11] JI YUE-PING, ZHANG YU-XIANG, LU XIAN-MING, et al. Study on prescription designed of the new type inhibitor. Chinese Journal of Explosives \& Propellants, 2000(4): 39-41.

[12] LIANG XI-YAO, REN YING-LIANG. Study on erosion reduction and useful properties of inhibitor. Ordnance Material Science and Engineering, 1997, 20(3): 24-29.

[13] BAI RUO-HUA. Application of erosion reducing additives as important technical measures in improving the erosion life of a gun barrel. Acta Armamentarii, 1995(3): 54-58.

[14] COTE P J, RICKARD C. Gas-metal reaction products in the erosion of chromium-plated gun bores. Wear, 2000, (241): 17-25.

[15] BRACUTI A J, FIELD R. Wear Reducing Additives. ARWEC-TR02006, New Jersey: U.S. Army Armament Research, Development and Engineering Center, 2002: 1-16.

[16] ZHEN SHUANG, LIU BO, LIU SHAO-WU, et al. Application of a new agent of low erosion in the small caliber weapon. Chinese Journal of Energetic Materials, 2011, 19(3): 335-338.

[17] CHEN YONG-CAI, SONG QIU-ZHI, WANG JIAN-ZHONG. Thermochemical erosion of propellant with nanometer additives. Acta Armamentarii, 2007, 28(3): 329-331.

[18] GUO DE-HUI, WU YONG-GANG. Research on the Anti-erosion Techniques of a Large-caliber Gun Barrel. Annual Meeting of China Ordnance Society, Suzhou, 2011: 178-183.

[19] YUN J, KIM H. Dual-responsive release behavior of $\mathrm{pH}$-sensitive PVA/PAAc hydrogels containing temperature-sensitive PVA/ PNIPAAm microcapsules. Polymer Bulletin, 2012(68): 1109- 1119.

[20] GALBIATI A, ROCCA B M, TABOLACCI C, et al. PVA engineered microcapsules for targeted delivery of camptothecin to HeLa cells. Materials Science and Engineering C, 2011(31): 1653-1659.

[21] LI J J, WANG B, YE G D, et al. Study of synthesizing energy storage microcapsules in PVA spinning solution and thermal regulating fibers prepared by this solution. Fibers and Polymers, 2013, 14(4): 537-541.

[22] YUN J, IM J S, LEE Y S, et al. pH and electro-responsive release behavior of MWCNT/PVA/PAAc composite microcapsules. Colloids and Surfaces A: Physicochemical and Engineering Aspects, 2010(368): 23-30.

[23] YAN JUN, WANG BIN, CUI HAI-PING, et al. Low temperature preparation and photo-absorbance property of micron size $\mathrm{Cu} /$ nano- $\mathrm{TiO}_{2}$ composite particles. Journal of Inorganic Materials, 2010, 25(4): 370-374.

[24] SONG C Y, YU W J, ZHAO B, et al. Efficient fabrication and photocatalytic properties of $\mathrm{TiO}_{2}$ hollow spheres. Catalysis Communications, 2009(10): 650-654.

[25] SONG QIU-ZHI, WANG JIAN-ZHONG, CHEN YONG-CAI, et al. Influence of dispersing property of nanomaterial mixed into propellant on the service life of gun barrel. Acta Armamentarii, 2009, 30(3): 289-294. 